Successive integrations by parts and application of the conditions $f^{m}(1)=0$ gives $I(x)=f(x)$.

The second solution can be verified in a similar way.

\title{
REFERENCES
}

1. R. G. Buschman, An inversion integral for a Legendre transformation, Amer. Math. Monthly 69 (1962), 288-289.

2. - An inversion integral, Proc. Amer. Math. Soc. 13 (1962), 675-677.

3. A. Erdélyi et al., Tables of integral transforms, Vol. 1, McGraw-Hill, New York, 1954.

4. - Higher transcendental functions, Vol. 2, McGraw-Hill, New York, 1954.

5. Ta Li, A new class of integral transforms, Proc. Amer. Math. Soc. 11 (1960), 290-298.

6. E. T. Titchmarch, Introduction to the theory of Fourier integrals, 2nd ed., Oxford Univ. Press, Oxford, 1959.

M. A. College of Technology, Bhopal (M.P.) India

\section{INFINITE ORDER DIFFERENTIAL EQUATIONS}

D. G. DICKSON

1. Introduction. Let $f(z)=\sum_{k=0}^{\infty} A_{k} z^{k}$ converge for $|z|<R$, where $0<R \leqq \infty$; let $E$ be the vector space of entire functions of exponential type less than $R$; and let $D=\sum_{k=0}^{\infty} A_{k} D^{k}$, where $D$ is the differential operator. The purpose of this paper is to provide a brief derivation of the results of Muggli [2, p. 154] regarding the general solutions in $E$ of the equations

$$
\begin{aligned}
& D \phi=0, \quad \text { and } \\
& D \phi=\psi .
\end{aligned}
$$

It will be shown that $D$ is a surjective endomorphism of $E$, reducing the problem of solving (2) to that of solving (1). It is easy to show that if $\zeta$ is a zero of $f$ of order at least $h+1$ and of modulus less that $R$, then $z^{h} e^{\xi z}$ is a solution of (1). If $B$ is the set of all such exponential monomials, then Muggli's result says that $B$ is a basis for the solutions of (1) and that each solution $\phi$ is representable as a sum of exponential monomials with exponent coefficients in the conjugate indicator diagram of $\phi$. Each solution of (2) is then representable as the sum of a contour integral and a solution of (1).

Results similar to these have been obtained by Sheffer [3, p. 255]

Received by the editors March 14, 1963. 
and Sikkema [4, p. 203]. In case $f$ itself is entire and of exponential type, the solutions of (1) are entire mean periodic functions and have been studied widely. The result concerning (1) may be viewed as a generalization of the fact that any function of exponential type $\sigma$ and of period $2 \pi$ can be expressed in the form $\sum c_{n} e^{i n z}$, where the sum is over integers $n$ with $|n| \leqq \sigma$. The explicitness of the result given here and its brief derivation are due to the use of a lemma which is perhaps of interest in itself.

2. Preliminaries. Let $C$ denote the disk $|z|<R$, and let $P^{\prime}$ denote the complement in the plane of the set $P$. For $\phi$ in $E, P(\phi)$ will denote the conjugate indicator diagram of $\phi$, and $L \phi$ will denote the Borel transform $[1$, p. 73] of $\phi$. Then $P(\phi) \subset C$, and $L \phi$ is analytic in $P^{\prime}(\phi)$. Also if $t \in C$, then $D_{z} e^{z t}=f(t) e^{z t}$, where $D_{z}=\sum_{k=0}^{\infty} A_{k} \partial^{k} / \partial z^{k}$.

The fact that $D$ maps $E$ into $E$ with $P(D \phi) \subset P(\phi)$ follows easily from the Pólya representation of $\phi[1$, p. 74]. For if $\gamma$ is a simple closed (rectifiable and positively oriented) curve in $C$ containing $P(\phi)$ in its interior, then

$$
\mathscr{D} \phi(z)=(2 \pi i)^{-1} \int_{\gamma} e^{z w} f(w) L \phi(w) d w,
$$

the change in order of integration and summation being justified by the uniform convergence of this integrand on $\gamma$. D $\phi$ is obviously entire, and since $\gamma$ may be chosen to be arbitrarily close to the boundary of $P(\phi)$, it easily follows that the indicator function of $D \phi$ is less than or equal to that of $\phi$ and so $P(D \phi) \subset P(\phi)$.

$D$ also maps $E$ onto $E$; in fact if $\psi \in E$, then there is a $\psi_{0}$ in $E$ such that $D \psi_{0}=\psi$ and $P\left(\psi_{0}\right)=P(\psi)$. For suppose that $\gamma$ is a simple closed curve in $C$ about $P(\psi)$ such that there are no zeros of $f$ on $\gamma$ or in the region common to the interior of $\gamma$ and $P^{\prime}(\psi)$. Let

$$
\psi_{0}(z)=\frac{1}{2 \pi i} \int_{\gamma} \frac{e^{z w} L \psi(w)}{f(w)} d w .
$$

Then $\psi_{0} \in E$ and since $\psi_{0}$ is independent of the choice of $\gamma$ for $\gamma$ in the zero free region mentioned, $P\left(\psi_{0}\right) \subset P(\psi)$. But easily $D \psi_{0}=\psi$, and since $P(\psi)=P\left(D \psi_{0}\right) \subset P\left(\psi_{0}\right), P\left(\psi_{0}\right)=P(\psi)$.

\section{Representation of solutions.}

Definition. If $\phi \in E$ and $t \in C$, then

$$
T \phi(t)=\left[D_{z} \int_{0}^{z} \phi(s) e^{(z-s) t} d s\right]_{z=0} .
$$


$T \phi$ is well defined; for if $t \in C$, then $e^{z t} \in E$ and its convolution with $\phi$ has as a type the maximum of $|t|$ and the type of $\phi$. Hence the convolution and its image under $D_{z}$ are in $E$. Further, $T \phi$ is analytic in $C$. For suppose $N$ is a closed circular neighborhood in $C$ and $t \in N$. Then the convolution of $\phi$ and $e^{z t}$ is of a type $\sigma<R$, and its Borel transform is $(z-t)^{-1} L \phi(z)$. By choosing a circle $\beta$ about the origin with radius between $\sigma$ and $R$ and which contains $N$ in its interior, we may write the convolution as a Pólya integral over $\beta$ and obtain

$$
T \phi(t)=\int_{\beta} H(w, t) d w, \text { where } H(w, t)=\frac{f(w) L \phi(w)}{2 \pi i(w-t)} .
$$

Application of Morera's theorem yields the analyticity of $T \phi$ in the interior of $N$.

Lemma. If $\phi \in E$ and $t \in C \cap P^{\prime}(\phi)$, then $T \phi(t)=f(t) L \phi(t)-L D \phi(t)$.

Proof. Let $\phi$ be of type $\sigma<R$ and $\epsilon=(R-\sigma) / 3$. Let $\alpha, \beta$, and $\gamma$ be the circles $|z|=\sigma+\epsilon,|z|=\sigma+2 \epsilon$, and $|z-(\sigma+R) / 2|=\epsilon / 3$, respectively. The left member of the identity to be established is analytic in $C$, while the right member is analytic in $C \cap P^{\prime}(\phi)$ since $P(D \phi) \subset P(\phi)$. Since both are uniform, it suffices to establish the identity for $t$ inside $\gamma$. Considering only such $t$, write $\phi(t)$ as a Pólya integral over $\alpha$, and then operate on this with $D$ and then with $L$, writing the latter as a Laplace transform from 0 to $\infty$. Then with $H(w, t)$ as in (4), $L D \phi(t)=-\int_{\alpha} H(w, t) d w$, the change in order of integration being justified by the observation that $\int_{0}^{\infty} \exp [(w-t) s] d s$ converges absolutely since $R(t-w)>0$ for $w$ on $\alpha$. Write $\int_{\alpha} H(w, t) d w$ as the difference of integrals over $\beta$ and $\gamma$. As in (4), the integral over $\beta$ is $T \phi(t)$ while the integral over $\gamma$ is $f(t) L \phi(t)$.

Definition. Let $\left\{\zeta_{k}\right\}_{k \in K}$ be the zeros of $f$ in $C$ with $m_{k+1}$ the order of $\zeta_{k}$. Let $c_{k}$ be a circle in $C$ about $\zeta_{k}$ containing no other zeros in or on itself. For each $k \in K$ and natural $h$, define the linear functional $\mathfrak{D}_{k h}$ on $E$ by

$$
\mathfrak{D}_{k h} \phi=\frac{1}{2 \pi i} \int_{c_{k}} \frac{\left(t-\zeta_{k}\right)^{h} T \phi(t)}{f(t)} d t .
$$

Using the lemma, it is easy to show that $\mathfrak{D}_{k h}\left(z^{p} \exp \zeta_{q} z\right)=h ! \delta_{p h} \delta_{q k}$ when $\zeta_{q}$ is a zero of $f$ of order at most $p+1$ and hence the elements in $B$ are linearly independent.

TheOREM. Let $\phi \in E$; then $D \phi=0$ if and only if

$$
\phi(z)=\sum_{k} e^{\zeta_{k} z} \sum_{h=0}^{m_{k}} \frac{\mathfrak{D}_{k h} \phi}{h !} z^{h},
$$


where $k$ ranges over those $k$ for which $\zeta_{k} \in P(\phi)$.

Proof. The fact that such a sum satisfies the equation is obvious. Suppose that $D \phi=0$. Upon writing the sum in (5) with the coefficients in integral form, the $m_{k}$ may be replaced by infinity since the terms so introduced are zero by Cauchy's theorem, and then the sum over $h$ may be replaced by an exponential function. The sum of the integrals over the $c_{k}$ may be replaced by one integral over a closed path $\gamma$ in $C$ about $P(\phi)$ whose interior contains no zeros of $f$ other than those in $P(\phi)$. Using the lemma and the fact that $D \phi=0$, the resulting integral is the Pólya representation of $\phi$.

COROllary. Let $\phi$ and $\psi \in E$; then $\mathscr{D} \phi=\psi$ if and only if

$$
\phi(z)=\psi_{0}(z)+\sum_{k} e^{\zeta_{k} z} \sum_{h=0}^{m_{k}} \frac{\mathfrak{D}_{k h}\left(\phi-\psi_{0}\right)}{h !} z^{h},
$$

where $k$ ranges over those $k$ for which $\zeta_{k} \in P\left(\phi-\psi_{0}\right)$ and $\psi_{0}$ is given by (3).

Other interesting results follow from the theorem and its corollary by observing their implications when $P(\phi)$ or $P\left(\phi-\psi_{0}\right)$ contains one or no zeros of $f$, as is the case when one of the diagrams is a point. It follows from the theorem that any solution of (1) in $E$ is actually the solution of a similar equation of finite order having a characteristic function dividing $f$ in the ring of functions analytic in $C$.

\section{REFERENCES}

1. R. P. Boas, Entire functions, Academic Press, New York, 1954.

2. H. Muggli, Differentialgleichungen unendlich hoher Ordnung mit konstanten Koeffizienten, Comment. Math. Helv. 11 (1938), 151-179.

3. I. M. Sheffer, Systems of linear differential equations of infinite order, with constant coefficients, Ann. of Math. (2) 30 (1928), 250-264.

4. P. C. Sikkema, Differential operators and differential equations of infinite order with constant coefficients, Noordhoff, Groningen, 1953.

UNIVERSity OF MichigaN 